\title{
Domination and Its Applications in Ad Hoc Wireless Networks with Unidirectional Links *
}

\author{
Jie $\mathrm{Wu}$ and Hailan $\mathrm{Li}$ \\ Department of Computer Science and Engineering \\ Florida Atlantic University \\ Boca Raton, FL 33431 \\ $\{$ jie, hli $\} @$ cse.fau.edu
}

\begin{abstract}
We consider an efficient distributed algorithm for determining a dominating and absorbant set of vertices (mobile hosts) in a given directed graph that represents an ad hoc wireless network with unidirectional links. This approach is based on the concept of dominating set in graph theory. A host $v$ is called a dominating neighbor (absorbant neighbor) of another host $u$ if there is a directed edge $(v, u)$ $((u, v))$. A subset of vertices is dominating and absorbant if every vertex not in the subset has one dominating neighbor and one absorbant neighbor in the subset. A quick formation process of a dominating and absorbant set is given and this set can be easily updated when the network topology changes dynamically. Ideas for dominating-set-based routing in an ad hoc wireless network with unidirectional links are also given. The effectiveness of the approach is confirmed through a simulation study.
\end{abstract}

\section{Introduction}

Recent advances in technology have provided portable computers with wireless interfaces that allow networked communication among mobile users. The resulting computing environment, often referred to as wireless mobile computing, no longer requires users to maintain a fixed and universally known position in the network and enables almost unrestricted mobility. An ad hoc wireless network [3] is a special type of wireless mobile networks in which a collection of mobile hosts with wireless network interfaces may form a temporary network, without the aid of any established infrastructure or centralized administration. The applications of ad hoc wireless networks range from civilian use (distributed computing, sensor networks) to disaster re-

* This work was supported in part by NSF grant CCR 9900646 and a grant from Motorola Inc. covery (search-and-rescue) and military use (battlefield). In the near future most of the commercial laptops and Personal Digital Assistants (PDAs) will be equipped with radios enabling them to form ad hoc "virtual" wireless networks.

Ad hoc wireless networks differ significantly from wired networks. The network topology may be quite dynamic Specifically, the network is dynamically self-organizing and self-configuring. In addition, resources such as bandwidth and batteries are scarce. When hosts are located closely together within wireless transmission range of each other, no real routing protocol is necessary. However, if two hosts that want to communicate are outside their wireless transmission ranges, they could communicate only if there are other hosts between them and can forward packets for them. In Figure 1, mobile hosts $\mathrm{A}$ and $\mathrm{C}$ are outside their transmission ranges (indicated by the circles around $\mathrm{A}$ and $\mathrm{C}$ ). If $A$ and $C$ wish to exchange packets, they may use host $B$ to forward packets for them, since B is within the transmission overlap of A's and C's ranges. In general, host connections in the ad hoc wireless network are determined based on their geographical distances in a 2-D or 3-D space and the corresponding graph is called a unit graph.

Routing is a process of sending a message from one mobile host in the network to another (it is also called unicast). Routing protocols for wireless networks normally call for mobility management and scalable design. Mobility management is done through information exchanges between moving hosts in the network. In general, when information exchanges occur frequently, the network maintains accurate information of host locations and other relevant information. However, frequent information exchanges can be costly, because they consume communication resources including bandwidth and power. With less frequent information exchanges, these costs diminish but there is more uncertainty about the host's location. Scalable design (one that works for large size networks) requires routing protocols and resource consumptions to be scalable. 


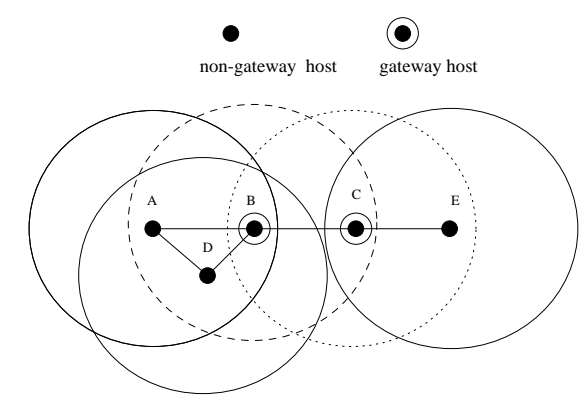

Figure 1. A sample ad hoc wireless network.

Routing in the ad hoc wireless network poses special challenges because of its infrastructureless network and its dynamic topology. The tunnel-based triangle routing of mobile IP [5] works well if there is a fixed infrastructure to support the concept of the "home agent". However, when all hosts move (including the home agent), such a strategy cannot be directly applied. Traditional routing protocols for wired networks that generally use either link state or distance vector [11] are no longer suitable for ad hoc wireless networks. In an environment with mobile hosts as routers, convergence to new, stable routes after dynamic changes in network topology may be slow and this process could be expensive due to low bandwidth. Mobility of hosts, which causes topological changes of the underlying network, also increases the volatility of network information. Routing information has to be localized to adapt quickly to changes such as host movements. In addition, the limitation of power leads users to connect/disconnect (also called switching on/off) mobile hosts frequently in order to reduce power consumption. This feature may also introduce more failures in mobile networks, which can be considered as a special form of mobility.

Various design choices are available for designing routing protocols for ad hoc wireless networks [8,9]; they are: (1) flat vs. hierarchical, (2) proactive vs. reactive, and (3) GPS-based vs. non-GPS-based. In a flat routing scheme, all hosts are treated equally, and therefore, any host can be used to forward packets between arbitrary sources and destinations. To permit scaling, hierarchical techniques are usually applied. The major advantage of hierarchical routing is the drastic reduction of routing table storage and processing overhead. In proactive routing, routes to all destinations are computed a priori and are maintained in the background via a periodic update process. In reactive routing, route to a specific destination is computed "on demand", i.e., only when needed. In non-GPS-based routing, routing process is based solely on the connections of hosts in the network. In GPS-based routing, each host knows its physical location by geolocation techniques such as Global Positioning System
(GPS). Routing is governed by physical distance between the source and destination. With few exceptions, such as dynamic source routing protocol (DSR) [1], most of existing protocols assume bidirectional links. Prakash [7] studied the impact of unidirectional links on some of the existing distance vector routing protocols such as destination sequenced distance vector (DSDV) [6], and found that unidirectional links prove costly for many existing protocols. It is shown that hosts need to exchange $O\left(n^{2}\right)$ information with each other in an $n$-node network.

In [12], we studied a special type of hierarchical routing based on the concept of dominating set [2] in graph theory. A subset of the vertices of a graph is a dominating set if every vertex not in the subset is adjacent to at least one vertex in the subset. A cluster consists of a dominating node $v$ together with a set of nodes that are not dominating nodes but are dominated by the dominating node $v$. In Figure 1, hosts $\mathrm{A}, \mathrm{B}$, and $\mathrm{D}$ form one cluster and hosts $\mathrm{C}$ and $\mathrm{E}$ form another one. $\mathrm{B}$ and $\mathrm{C}$ are dominating nodes, also called gateway hosts, which are connected. A, D, and E are called non-gateway hosts. A special procedure, called the marking process, is proposed that can quickly identify a set of connected dominating set. The main features of the marking process lie in its simplicity and effectiveness, making it more suitable for dynamic networks without introducing much overhead. The reduced graph is a subgraph induced from the connected dominating set. Based on the concept of domination, each non-dominating node has a dominating neighbor. Therefore, the routing process can be restricted to the reduced graph with the first hop (last hop) being a hop from a non-dominating node (which is a source) to a dominating node in the reduced graph (a dominating node to a non-dominating node (destination)) if needed. The main advantage of dominating-set-based routing is that it simplifies the routing process to the one in a smaller subnetwork generated from the connected dominating set, which means that only gateway hosts need to keep routing information. Other dominating-set-based routing exist $[4,10]$; however, these methods need a non-constant number of rounds to determine a (connected) dominating set.

In this paper, we extend the dominating-set-based routing to ad hoc wireless networks with unidirectional links. In an ad hoc wireless network, some links may be unidirectional due to the hidden terminal problem [11]. For example, $\mathrm{E}$ in Figure 1 can receive signals from $\mathrm{C}$, but $\mathrm{C}$ may not be able to receive signals from $\mathrm{E}$ due to the interference of signals sent from B. In this case, the connection between $\mathrm{E}$ and $\mathrm{C}$ is unidirectional (at least temporarily). An ad hoc wireless network is represented as a directed graph, $D=(V, A)$, consisting of a finite set $V$ of vertices and a set $A$ of directed edges. A host $v$ in $V$ is called a dominating neighbor (absorbant neighbor) of another host $u$ in $V$ if there is a directed link $(v, u)((u, v))$. A subset of ver- 
tices (mobile hosts) is dominating and absorbant if every vertex not in the subset has one dominating neighbor and one absorbant neighbor in the subset. A special procedure called the extended marking process is proposed. This process needs only 2- or 3-round of information exchanges to determine a connected dominating and absorbant set. We can also apply the extended marking process repeatly to form a hierarchy of dominating sets. The effectiveness of the extended marking process, in terms of finding a small dominating and absorbant set, is verified through a simulation study. Ideas for dominating-set-based routing in the ad hoc wireless network are also described. Throughout the paper, we use the terms: host, node, and vertex interchangeably; link and edge interchangeably.

The paper is organized as follows: Section 2 presents the extended marking process to calculate a small dominating and absorbant set in a directed graph. Several implementation issues are also discussed. Dominating-set-based routing is discussed in Section 3. Hierarchical formation of dominating and absorbant sets and ideas for hierarchical routing are discussed. Ways to update the dominating and absorbant set when the network topology changes dynamically are also presented. Section 4 shows simulation results and the paper concludes in Section 5.

\section{Domination in Directed Graphs}

The directed graph $D$ is a simple graph without self-loop and multiple edges. A directed (also called unidirectional) edge from $u$ to $v$ is denoted by an ordered pair $(u, v)$. If $(u, v)$ is an edge in $D$, we say that $u$ dominates $v$ and $v$ is an absorbant of $u$. A set $V^{\prime} \subset V$ is a dominating set of $D$ if every vertex $v \in V-V^{\prime}$ is dominated by at least one vertex $u \in V^{\prime}$. Also, a set $V^{\prime} \subset V$ is called an absorbant set if for every vertex $u \in V-V^{\prime}$, there exists a vertex $v \in V^{\prime}$ which is an absorbant of $u$. The dominating neighbor set of vertex $u$ is defined as $\{w:(w, u) \in A\}$. The absorbant neighbor set of vertex $u$ is defined as $\{v:(u, v) \in A\}$. A directed graph $D$ is strongly connected if for any two vertices $u$ and $v$, both $(u, v)$-path (i.e., a path connecting $u$ to $v$ ) and $(v, u)$-path exist. Throughout the paper, it is assumed that $D$ is strongly connected; otherwise, the network management subsystem will partition the network into a set of independent subnetworks, each of which is strongly connected.

Extended marking process. To determine a set that is both dominating and absorbant, we propose the following extended marking process. $m(u)$ is a marker for vertex $u \in V$, which is either $\mathrm{T}$ (marked) or F (unmarked).

1. Initially assign $\mathrm{F}$ to each $u \in V$.

2. $u$ changes its marker $m(u)$ to $\mathrm{T}$ if there exist vertices

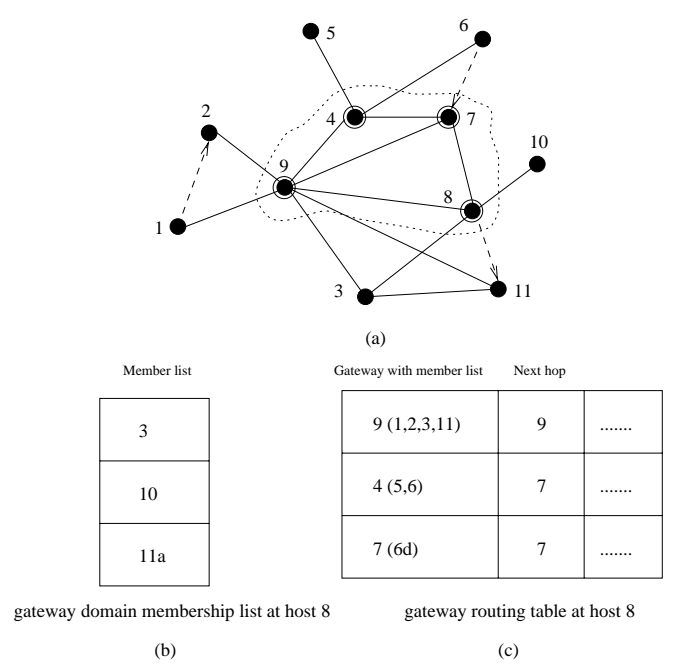

\section{Figure 2. (a) Another sample network with (b) gateway domain membership list and (c) gate- way routing table.} $v$ and $w$ such that $(w, u) \in A$ and $(u, v) \in A$, but
$(w, v) \notin A$.

Figure 2 (a) shows four gateway hosts 4, 7, 8, and 9 derived from the extended marking process. Arrow dashed lines correspond to unidirectional edges and solid lines represent bidirectional edges. A bidirectional edge $\{v, u\}$ can be considered as two unidirectional edges $(v, u)$ and $(u, v)$. Note that the above extended marking process requires each vertex $u$ to know only its absorbant neighbor set. Figure 3 shows three assignments of $u$, with one dominating neighbor $w$ and one absorbant neighbor $v$. The only case in Figure 3 with $m(u)=F$ is when $(w, v) \in A$, for every dominating neighbor $w$ and every absorbant neighbor $v$ of $u$. The fourth case, where $v$ and $w$ are bidirectionally connected, is not shown. Assume that $V^{\prime}$ is the set of vertices that are marked $\mathrm{T}$ in $V$, i.e., $V^{\prime}=\{u: u \in V, m(u)=T\}$. The reduced graph $D^{\prime}$ is the subgraph of $D$ induced by $V^{\prime}$, i.e., $D^{\prime}=D\left[V^{\prime}\right]$.

Theorem 1: Given a $D=(V, A)$ that is strongly connected, the vertex subset $V^{\prime}$, derived from the extended marking process, has the following properties: (1) $V^{\prime}$ is empty if and only if $D$ is completely connected, i.e., for every pair of vertices $u$ and $v$, there are two edges $(u, v)$ and $(v, u)$. (2) If $D$ is not completely connected, $V^{\prime}$ forms a dominating and absorbant set.

Proof: Arbitrarily select two vertices $u$ and $v$ from $D=$ $(V, A)$. If $u$ and $v$ are bidirectionally connected, i.e. $(u, v) \in A$ and $(v, u) \in A$ exist, neither $u$ nor $v$ will be marked $T$. That is, $V^{\prime}$ is empty when $D$ is completely con- 
nected. If $D$ is not completely connected, select two vertices $v$ and $u$ such that $(v, u) \notin A$. Since $D$ is strongly connected, we can find a shortest path $\left(v, \ldots, u_{2}, u_{1}, u\right)$ from $v$ to $u$. Clearly, $\left(u_{2}, u_{1}\right) \in A$ and $\left(u_{1}, u\right) \in A$, but $\left(u_{2}, u\right) \notin A$. Based on the extended marking process, $u_{1}$ is marked T, and hence, $V^{\prime}$ is not empty. In other words, if $V^{\prime}$ is empty $D$ must be complete connected.

Assume that $D$ is not completely connected. Arbitrarily select vertex $u$ such that $m(u)=F$. If such a vertex does not exist, the theorem is proved. Select another vertex $v$ such that $m(v)=T$. If such a $v$ does not exist, then $V^{\prime}$ is empty and $D$ is complete connected, a contradiction. Construct a shortest path $\left(v, \ldots, u_{1}, u\right)$, based on the extended marking process, $m\left(u_{1}\right)=T$. (When $(v, u) \in A, u_{1}$ is $v$.) Therefore, $u$ has a dominating neighbor $u_{1}$. Similarly, constructing a shortest path $\left(u, u_{1}, \ldots, v\right)$ from $u$ to $v$, we can prove that $u_{1}$ (an absorbant neighbor of $u$ ) is marked $T$.

When the given $D$ is completely connected, all vertices are marked $F$. This make sense, because if all vertices are directly connected, there is no need to use dominating and absorbant set to reduce $D$.

Theorem 2: $V^{\prime}$ includes all the intermediate vertices of any shortest path.

Proof: We prove this theorem by contradiction. Assume that a shortest path between two vertices $v$ and $u$ includes an unmarked vertex $v_{i}$ as an intermediate vertex, in other words, this path can be represented as $\left(v, \ldots, v_{i-1}, v_{i}, v_{i+1}, \ldots, u\right)$. Because $m\left(v_{i}\right)=F$, there must be a connection from $v_{i-1}$ to $v_{i+1}$ based on the extended marking process. Therefore, a shorter path between $v$ and $u$ can be found as $\left(v, \ldots, v_{i-1}, v_{i+1}, \ldots, u\right)$. This contradicts the original assumption.

Theorem 3: The reduced graph $D^{\prime}=D\left[V^{\prime}\right]$ is a strongly connected graph.

Proof: Randomly select two vertices $v$ and $u$ in $D^{\prime}$, assume that $\left(v, v_{1}, v_{2}, \ldots, v_{k}, u\right)$ is a shortest path from $v$ to $u$ in $D$. This path is also a path in $D^{\prime}$ based on Theorem 2.

Theorems 1, 2, and 3 serve as bases of the dominatingset-based routing. The dominating and absorbant set derived from the extended marking process has the desirable properties of routing optimality (Theorem 2 ) and connectivity (Theorem 3). However, in general the derived dominating and absorbant set is not minimum.

Extensions. Two rules are proposed to reduce the size of a connected dominating and absorbant set generated from the extended marking process. We first assign a distinct label, $i d(v)$, to each vertex $v$ in $V$. In a directed graph, $N_{d}(u)$ $\left(N_{a}(u)\right)$ represents the dominating (absorbant) neighbor set. The neighbor set is the union of the corresponding dominating neighbor and absorbant neighbor sets, i.e., $N(u)=N_{a}(u) \cup N_{d}(u)$. Vertex $u$ is called neighbor of

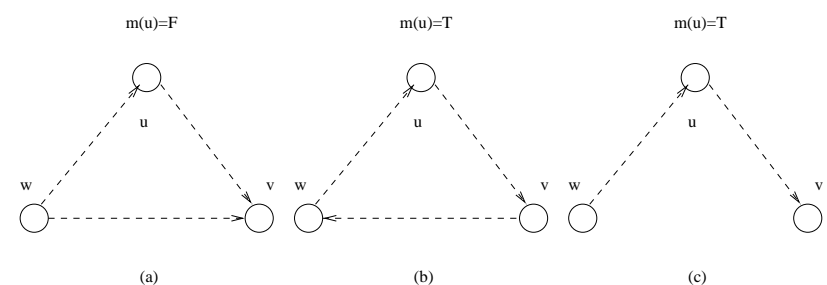

\section{Figure 3. Markers for three different situa- tions.}

vertex $v$ if $u$ is a dominating, absorbant, or dominating and absorbant neighbor of $v$.

Rule 1: Consider two vertices $u$ and $v$ in reduced graph $D^{\prime}$. Unmark u, i.e., $D^{\prime}$ is changed to $D_{u}^{\prime}=D^{\prime}-\{u\}$, if the following conditions hold: (1) $N_{d}(u)-\{v\} \subseteq N_{d}(v)$ and $N_{a}(u)-\{v\} \subseteq N_{a}(v)$ in D. (2) $i d(u)<i d(v)$.

The above rule indicates when the dominating (absorbant) neighbor set of $u$ (excluding $v$ ) is covered by the dominating (absorbant) of $v$, vertex $u$ can be removed from $D^{\prime}$ if $u$ 's $i d$ is smaller than $v$ 's. Note that $u$ and $v$ may or may not be connected (bidirectional or unidirectional). The role of id is very important to avoid "illegal simultaneous" removal of vertices in $V^{\prime}$ when Rule 1 is applied "simultaneously" to each vertex. In general, vertex $u$ cannot be removed even if $N_{d}(u)-\{v\} \subseteq N_{d}(v)$ and $N_{a}(u)-\{v\} \subseteq N_{a}(v)$ in $D$, unless $i d(u)<i d(v)$. Consider a graph of four vertices, $u, v, s$, and $d$, with four undirected edges $\{u, s\},\{s, v\},\{v, d\}$, and $\{d, u\}$. All four vertices will be marked using the extended marking process. Also, $N_{d}(u)=N_{d}(v)=N_{a}(u)=N_{a}(v)=\{s, t\}$ $\left(N_{d}(s)=N_{d}(t)=N_{a}(s)=N_{a}(t)=\{u, v\}\right)$. Without $i d$, both $u$ and $v$ (also $s$ and $t$ ) will be unmarked, leaving no marked vertex. With $i d$, one of $u$ and $v$ (also $s$ and $t$ ) will be unmarked, leaving two marked vertices.

Rule 2: Assume that $u, v$ and $w$ are three marked vertices in $D^{\prime}$. Unmark $u$ if the following conditions hold: (1) $N_{d}(u)-$ $\{v, w\} \subseteq N_{d}(v) \cup N_{d}(w)$ and $N_{a}(u)-\{v, w\} \subseteq N_{a}(v) \cup$ $N_{a}(w)$ in D. (2) $i d(u)=\min \{i d(u), i d(v), i d(w)\}$. (3) $v$ and $w$ are bidirectionally connected.

The above rule indicates that when the dominating (absorbant) neighbor set of node $u$ (excluding $v$ and $w$ ) is covered by the union of dominating (absorbant) sets of $v$ and $w$, vertex $u$ can be removed from $D^{\prime}$ if $u$ 's $i d$ is smaller than $v$ 's and $w$ 's. Again, $u$ and $v(w)$ may or may not be connected.

Figure 4 shows an example of using the extended marking process and its extensions (two rules) to identify a set of connected dominating and absorbant nodes. Figure 4 (a) shows the gateway nodes (nodes with double circles) de- 


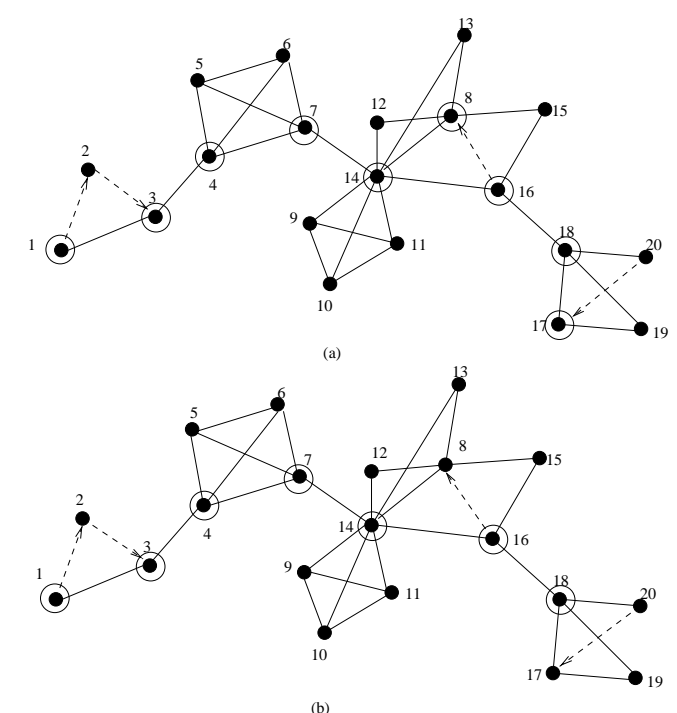

Figure 4. Marked gateways (a) by using the extended marking process and (b) by applying Rules 1 and 2.

rived by the extended marking process without applying two rules. Figure 4 (b) shows the remaining gateway nodes after applying two rules.

Assume that $V_{*}^{\prime}$ is the resultant dominating and absorbant set when Rule 1 and Rule 2 are simultaneously applied to all vertices in $V^{\prime}$. The following result shows that $V_{*}^{\prime}$ (its induced graph is $D_{*}^{\prime}$ ) is still a connected dominating and absorbant set of $V$. The shortest path property of Theorem 3 still holds in $D_{*}^{\prime}$ for Rule 1, but not for Rule 2 .

Theorem 4: If $V^{\prime}$ is a strongly connected dominating and absorbant set of $D$ derived by using the extended marking process, then $V_{*}^{\prime}$ derived by using Rule 1 and Rule 2 on all vertices in $V^{\prime}$ is still a strongly connected dominating and absorbant set of $V$. In addition, if $V_{*}^{\prime}$ is derived by applying Rule 1 alone, then $V_{*}^{\prime}$ still includes all intermediate vertices of at least one shortest path for any pair of vertices in $V$.

The proof for Theorem 4 is length and is omitted here. For each application of Rule 2, the length of a shortest path (that includes $u$ as an intermediate node) increases by at most one.

Implementation issues. To provide a decentralized implementation, each host keeps a list of its neighbors and sends this list to all its neighbors. By doing so each host has distance-2 neighborhood information, i.e., information about its neighbors and the neighbors of all its neighbors. The extended marking process is then applied to each individual host to determine its status. The gateway and nongateway status (i.e., marked and unmarked status) of hosts are exchanged among the neighboring hosts. In this way, each non-gateway host $u$ knows the gateway host within each cluster that contains $u$. Similarly, each gateway $u$ knows its non-gateway neighbors within the cluster that contains $u$. In an ad hoc wireless network, it would not be cost-effective for each host to keep distance- $k$ neighborhood information, where $k$ is a relatively large integer corresponding to a distance. However, distance- 2 neighborhood information forces a restricted implementation of Rules 1 and 2. Specifically, it requires $u$ and $v$ to be neighbors (actually they should be bidirectionally connected) in Rule 1 and $v$ and $w$ are neighbors of $u$ in Rule 2 .

The existence of unidirectional links brings a special challenge: If a link is directed from host $u$ to host $v$, host $v$ can receive packets from host $u$, but host $u$ is not aware of the existence of $v$. One possible solution is that each host emits a beacon at regular intervals to its neighbors. Once host $u$ receives a beacon containing its $i d$ and $i d$ of $v$ as its immediate forwarding host, host $u$ knows host $v$ as an absorbant neighbor. Note that the beacon is also used to transit neighborhood information of $v$ to $u$. Certainly, pure broadcasting of beacon through flooding is not feasible because of its high consumption of bandwidth and energy. Each host has to selectively emit and forward a beacon. For example, if there is a bidirectional link between $u$ and $v, v$ only needs to send back an incoming beacon from $u$ and there is no need to broadcast it to other neighbors. However, $u$ does not know in advance the existence of link $(u, v)$ to avoid broadcasting.

To avoid global broadcasting (also called flooding), we can add a Time-To-Live (TTL) field to limit the number of hops a beacon is allowed to travel. In the above case, TTL is set to 1 (which corresponds to distance-2 neighborhood information). In general, in order to recognize all unidirectional links, TTL should be set to the maximum cycle number of all links in $D$, where the cycle number of a link is defined as the size of the shortest cycle containing the link in $D$ minus one. Clearly, in an undirected graph, the size of the shortest cycle for each link is 2 , and hence, the maximum cycle number is 1 . In a directed graph, TTL is usually set to a small number, say 2 or 3 , to save bandwidth and energy. As a result, reachability information may not be able to be propagated back to some senders that have unidirectional outgoing links and the corresponding links are dropped. Therefore, an intelligent decision on TTL is needed to balance the bandwidth and energy consumption and the drop rate of unidirectional links. Figures 5 (a) and (b) show two possible ways for host $u$ to recognize an absorbant neighbor $v$ when TTL is set to 2 . Arrow dotted lines correspond to links used to pass a beacon.

The selection of TTL=2 (or 3) broadens the application scope of the original implementation (based on distance-2 neighborhood information) of Rules 1 and 2. For example, 


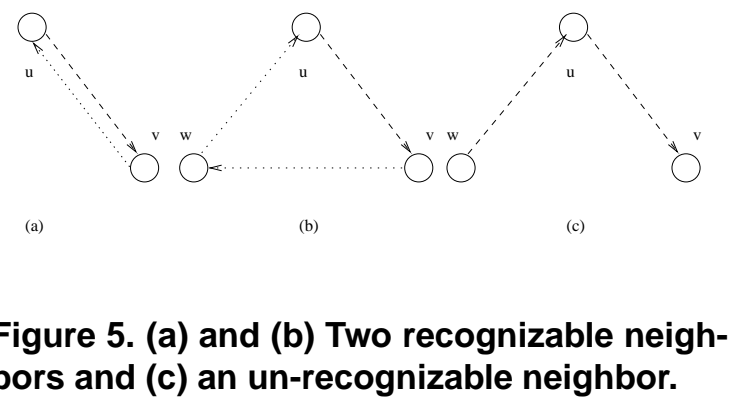

it is not required that $u$ and $v$ in Rule 1 to be neighbors, as long as they stay within 2-hop apart and can receive the neighbor set of each other. Again consider the graph of four vertices, $u, v, s$, and $d$, with four undirected edges $\{u, s\},\{s, v\},\{v, d\}$, and $\{d, u\}$. All four vertices will be marked using the extended marking process. Although $u$ and $v$ (similarly for $s$ and $d$ ) are not directly connected, each can receive the beacon (containing neighbor set information) sent from the other when TTL $=2$, one of $u$ and $v$ (also one of $s$ and $d$ ) can be unmarked by applying Rule 1.

Based on the above analysis, a node can recognize and receive information from its dominating neighbors. The problem lies in its absorbant neighbors. For example, suppose node $u$ has only two connections with $v$ and $w:(w, u)$ and $(u, v)$. In addition, $v$ and $w$ are the only two neighbors of $u$. We consider the following four cases: (1) If there is a bidirectional link between $v$ and $w$, then the beacon and the neighborhood information from $v$ will be passed to $u$ via $w$. Therefore, $u$ can correctly assign its mark to T. (2) If there is a unidirectional link from $v$ and $w$ (see Figure 5 (b)), then the situation is similar to the first case and $u$ can correctly assign its mark to T. (3) If there is a unidirectional link from $w$ to $v, u$ should be assigned F. Since the default value of $m(u)$ is assigned $\mathrm{F}$, this result is correct by default, even though $u$ does not have connectivity information. (4) If there is no connection between $v$ and $w, u$ should be marked $\mathrm{T}$ (see Figure 5 (c)). In this case, neighborhood information of $v$ should be passed to either $u$ or $w$ via a fourth node $v_{a}$. Note that path $\left(v, v_{a}, w, u\right)$ requires TTL to be 3 while path $\left(v, v_{a}, u\right)$ requires TTL to be only 2 .

Case (4) above may cause problems when absorbant neighbor $v_{a}$ of $v$ (connecting to $u$ or $w$ ) does not exist. One possible solution is to assign the default value to $\mathrm{T}$ for all vertices initially. The mark of a vertex $u$ can be changed to $\mathrm{F}$ only if the conditions in the extended marking process hold for all pairs of $u$ 's neighbors: $v$ and $w$; that is, $m(u)=F$ if $(w, u),(u, v)$, and $(w, v)$ exist. By doing this, case 4 is covered. However, in case 3 vertex $u$ will be marked $\mathrm{T}$, rather than $\mathrm{F}$, generating a larger dominating and absorbant set. Note that it is better to include more vertices in the set than to miss one.

\section{Dominating-Set-Based Routing}

Dominating-set-based process. The routing process in a dominating-set-based routing is usually divided into three steps: (1) If the source is not a gateway host, it forwards packets to a source gateway, which is one of the absorbant gateway hosts. (2) This source gateway acts as a new source to route packets in the reduced graph generated from the connected dominating set. (3) Eventually, packets reach a destination gateway, which is either the destination host itself or a gateway of the destination host, i.e., it is a dominating neighbor of the destination. In the latter case, the destination gateway forwards packets directly to the destination host. Note that the distributed formation of each cluster can be easily done by exchanging markers between neighbors.

We use distance-vector-type routing to illustrate the dominating-set-based routing. Each gateway host keeps the following information: gateway domain membership list and gateway routing table. The gateway domain membership list of a gateway host is a list of non-gateway hosts that are adjacent to the gateway host. The gateway routing table at gateway host $v$ includes one entry for each gateway host other than $v$, together with its domain membership list.

Given an ad hoc wireless network as shown in Figure 2, Figure 2 (a) shows four gateway hosts 4, 7, 8, and 9 derived from the extended marking process. Figure 2 (b) shows that host 8 has three members $3,10,11_{a}$ in its gateway domain membership list. A neighbor with a subscript $a(d)$ corresponds to an absorbant neighbor (dominating neighbor). Figure 2 (c) shows the gateway routing table at host 8 . The first column of Figure 2 (c) is a set of entries for each destination gateway (excluding host 8 ) together with its membership list. The second column of Figure 2 (c) shows next hop information of a shortest path (the length of a path is measured by hop count). Other columns of this table, including distance, are not shown. Note that the way that routing tables are constructed and updated in the reduced graph can be different from protocols to protocols. Potentially, many existing routing protocols: proactive, reactive, non-GPS-based, GPS-based, or their combinations can be used as underlying protocols for the reduced graph.

Hierarchical structure. Hierarchical routing aggregates hosts into clusters and clusters into superclusters, and so on. If addresses of the destination host and the host that is forwarding the packet belong to different super-clusters, then forwarding will be done with an inter super-cluster route; if they belong to the same supercluster but to different clusters, forwarding will be done using inter cluster routes; if they belong to the same cluster, forwarding will be done by using intra cluster routes.

The extended marking process can be applied to the reduced graph to generate a dominating set of the dominating set (the resultant graph forms a super-cluster). In this way, 
we can define a hierarchy of networks, with the original network being at level 1 , the reduced graph induced from the dominating set being at level 2 , and so on. To evaluate the effectiveness of the extended marking process in obtaining a dominating set from a given unit graph, we introduce a concept of dominating ratio (domi_ratio) which is the ratio of the size of the resultant dominating set and the size of the original network. Clearly, $0<$ domi_ratio $\leq 1$. A small domi_ratio corresponds to a small dominating set. Unfortunately, the minimum dominating ratio is not known a priori. There are several lower bounds [2] of dominating ratio for graphs of different properties and these bounds can be used as references of comparison. In Figure 2, the domi_ratio at level 1 is $4 / 11$ (four dominating nodes out of a total of eleven hosts in the network) and the domi_ratio at level 2 is $2 / 4$.

One critical issue in the design of a hierarchical structure is to decide an appropriate level of hierarchy. The extended marking process is said to be ineffective for a given network if the corresponding dominating ratio is close to 1 or above a given threshold. A threshold can be defined in such a way that the benefit from the reduction of the network overweights the cost of maintaining an extra level of hierarchy. If the extended marking process is applied repeatly on the resultant graph (induced from the dominating set) until it is no longer effective, the corresponding level is called maximum hierarchical level. Implementing hierarchical routing in a highly dynamic network requires sound solutions of several issues. Other than the dynamic formation of hierarchy, routing protocols must adapt to changes in hierarchical connectivity as well as to changes their connections to other mobile hosts.

Update/recalculation. In the ad hoc wireless network, each host can move around without speed and distance limitation. Also in order to reduce power consumption, mobile hosts may switch off at any time and switch on later. We can summarize topological changes of an ad hoc wireless network into three different types: mobile host switching on, mobile host switching off, and mobile host movement. The challenge here is when and how each host should update/recalculate gateway information. The gateway update means that only individual mobile hosts update their gateway status. The gateway recalculation means that the entire network recalculates gateway/non-gateway status. If many mobile hosts in the network are in movement, gateway recalculation might be a better approach, i.e., the dominating and absorbant set is recalculated from scratch. On the other hand, if only few mobile hosts are in movement, then gateway information can be updated locally.

When a mobile host $u$ switches on, only its non-gateway neighbors, along with host $u$, need to update their status, because any gateway neighbor will still remain as gateway after a new vertex $u$ is added. For example, in Figure 6

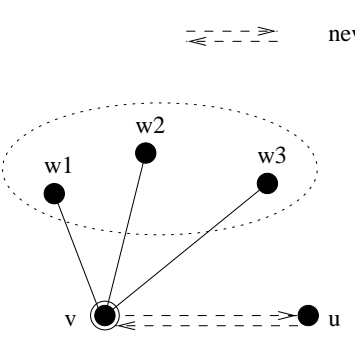

(a) gateway neighbor $\mathrm{u}$

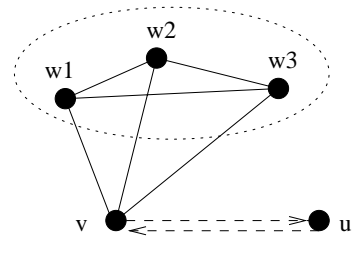

(b) non-gateway neighbor $\mathrm{w}$
Figure 6. Mobile host $u$ switching on.

(a), when host $u$ switches on, the status of gateway neighbor $v$ is not affected, because at least two of $v$ 's neighbors $w_{1}, w_{2}$, and $w_{3}$ are not connected originally and these connections will not be affected by host $u$ 's switching on. On the other hand, in Figure 6 (b), host $u$ 's switch on might lead non-gateway neighbor host $v$ to mark itself as gateway, depending on the connection between host $u$ and $v$ 's neighbors $w_{1}, w_{2}$, and $w_{3}$. Note that a new link can be either unidirectional or bidirectional. The corresponding update process for the case of a mobile host $u$ switching on is the following:

1. Mobile host $u$ broadcasts to its neighbors about its switching on. In particular, a $k$-hop beacon is used to broadcast this signal to its dominating neighbors.

2. Each host $v \in u \cup N(u)$ exchanges its neighbor set $N(v)$ with its neighbors. Again, a $k$-hop beacon is needed for the exchange.

3. Host $u$ assigns its marker $m(u)$ to $T$ if there are $(v, u) \in A$ and $(u, w) \in A$, but $(v, w) \notin A$.

4. Each non-gateway host $v \in N(u)$ assigns its marker $m(v)$ to $T$ if $(w, v) \in A$ and $(v, u) \in A$, but $(w, u) \notin$ $A$; or $(u, v) \in A$ and $(v, w) \in A$, but $(u, w) \notin A$.

5. Whenever there is a newly marked gateway, host $u$ and all its gateway neighbors apply Rules 1 and 2 to reduce the number of gateway hosts.

The case for a host switching off is similar to the one for a host switching on. A mobile host $u$ 's movement can be viewed as several simultaneous or non simultaneous link connections and disconnections. For example, when a mobile host moves, it may lead several link disconnections with its neighbor hosts, and at the same time, it may have new link connections to the hosts within its wireless transmission range, these new links may be disconnected again depending on the way host $u$ moves. In order to synchronize mobile host's movement in gateway updates, just before mobile host $u$ starts to move, it sends out a special sig- 

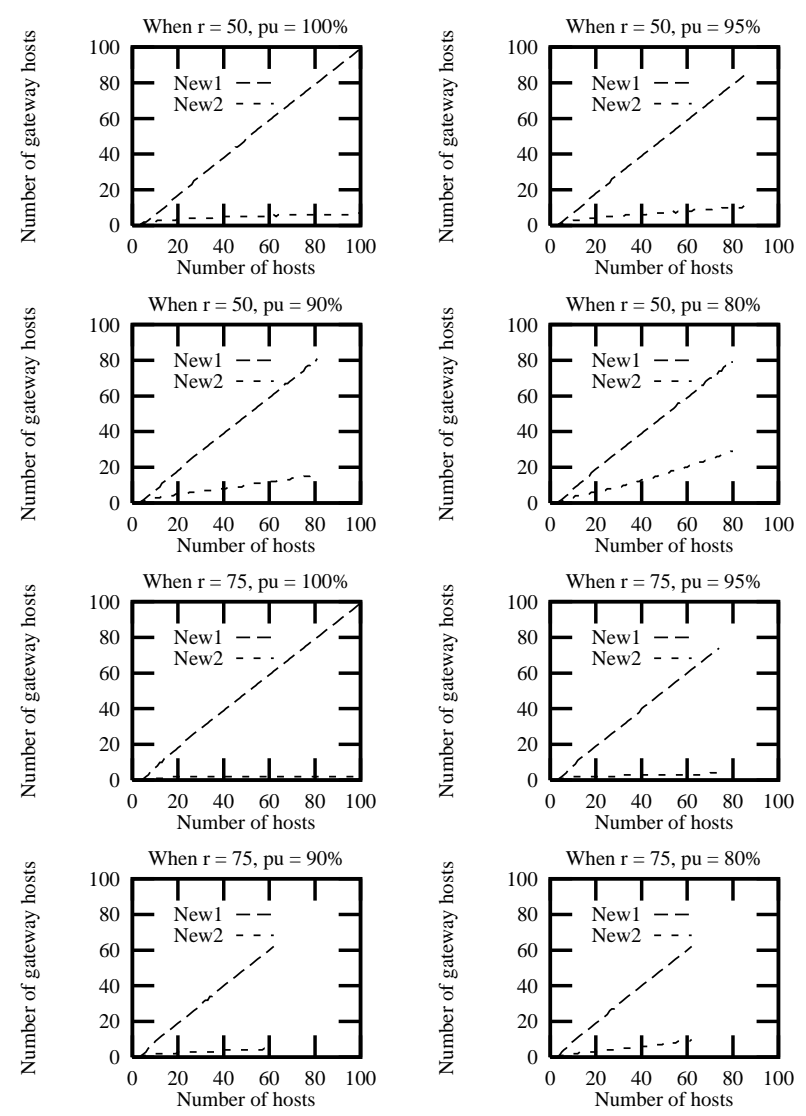

\section{Figure 7. Average number of gateway hosts} relative to the number of hosts $\nu$.

nal $\{\operatorname{id}(u)$, start $\}$, then during its movement host $u$ continuously sends out signal $\{\operatorname{id}(u)$, heart_beat $\}$ at every $\tau$ time interval, and when it stops moving around, host $u$ sends out signal $\{\operatorname{id}(u)$, stop $\}$. The details of the approach are similar to the one for undirected graphs [12].

\section{Simulation}

We have conducted a simulation study to measure the size of the dominating and absorbant set generated from the extended marking process. Three sets of simulation are conducted: (1) The effectiveness of Rules 1 and 2 are evaluated. Simulation is based on the restricted implementation using distance-2 neighborhood information. Four sets of data are used corresponding to four different percentages of undirected links $(p u)$ : 100\% (where all links are undirected), $95 \%, 90 \%, 80 \%$. (2) The drop rates of unidirectional links are collected for (a) 2-hop beacon and (b) 3-hop beacon, respectively. Again, the percentages of undirected links are $95 \%, 90 \%, 80 \%$. (3) The maximum hierarchical level is calculated where the threshold (for ineffectiveness) is set to be 1 . That is, the marking process terminates when the resultant graph is the same as the original graph. In this simulation, it is assumed that all links are undirected.

The simulation was performed using the following parameters: $\nu$ represents the number of mobile hosts in the network, $\gamma$ the number of gateways (the size of the dominating set), $r$ the radius of mobile host's transmission range, $p u$ the percentage of undirected links, $d r_{2}$ and $d r_{3}$ the drop rates of unidirectional links when 2-hop beacon and 3-hop beacon are used, respectively, and New1 (New2) the number of gateway hosts calculated by extended marking process without using two rules (with applying two rules).

Random graphs are generated in a $100 \times 100$ square units of a 2-D plane, by randomly throwing a certain number of mobile hosts. A 2-D plane resembles an actual ad hoc wireless network where mobile hosts usually stay on ground. Each mobile host has the same transmission radius $r$. If the distance between any two hosts is less than radius $r$, there is a link connection between these two hosts. A random number, between 0 and 100 inclusive, is associated with each link. If the random number is less than or equal to $p u$, then the corresponding link is undirected; otherwise, it is directed. In each simulation, the radius of the mobile host's transmission area $r$ is set to two different values: 50 and 75. In this way, we can control the density of random graphs, since the density of random graphs increases as $r$ increases. Each random graph must be a strongly connected graph; otherwise, it is discarded. For each combination of $r$ and $p u$, we also vary the number of mobile hosts $\nu$ from 0 to 100 . For each $\nu$, the random graph is generated 1000 times. All simulation results are measured by simply taking the average of all cases.

Figure 7 shows the number of gateways versus the number of hosts in the network for the increasing number of hosts. By applying two rules, the performance of our approach (the curve New2) improves dramatically over the one without using two rules (the curve New1). Also, as the percentage of undirected links drops, the average number of gateway hosts increases, especially for the extended marking process with two rules. Figure 8 shows the average drop rates of unidirectional links. The results show that the drop rates are small in all cases, especially for 3-hop beacons where the rates are close to 0 . Therefore, 2-hop and 3-hop beacons are sufficient to detect unidirectional links as long as $p u$ (the percentage of undirected links) stays relatively high. Figure 9 shows the maximum hierarchical level relative to the number of hosts in the network for different radii $r$. We can see that the maximum hierarchical level ranges from 3 to 6 when the radii of mobile host's transmission ranges are neither too small nor too large, say, $r=25$ or 50 . On the other hand, when $r=75$, the average maximum hierarchical level is close to 1 . Therefore, the hierarchi- 

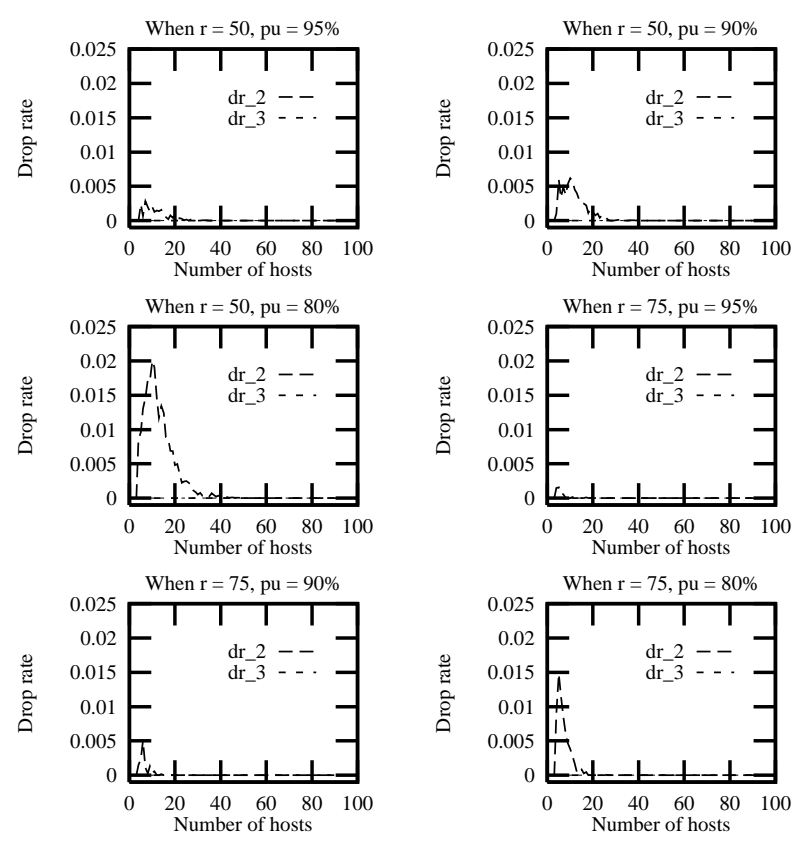

\section{Figure 8. Average drop rate of unidirectional links.}

cal approach is ineffective for networks with relatively large mobile transmission radii.

\section{Conclusions}

In this paper, we have extended the dominating-set-based routing in ad hoc wireless networks with unidirectional links. This approach is based on finding a core which is a dominating and absorbant set in a directed graph representing the topology of the ad hoc wireless network. An extended marking process has been presented that can identify such a core quickly and can update it easily in a dynamic environment. Although it is unlikely that the dominatingset-based routing can solve all the critical issues in routing in the ad hoc wireless network, we believe that it offers a very promising and unique combination of several of existing approaches in conjunction with the novel use of the dominating and absorbant set. Our future work will focus on working out design details of dominating-set-based routing and verify the effectiveness of the design through an extensive simulation study.

\section{References}

[1] J. Broch, D. Johnson, and D. Maltz. The dynamic source routing protocol for mobile ad hoc networks. IETF, Internet Draft, draft-ietf-manet-dsr00.txt, March 1998.

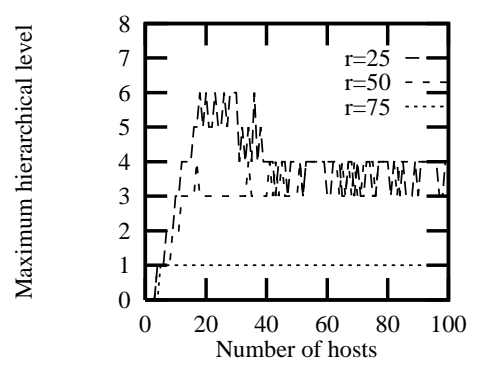

Figure 9. The maximum hierarchical level relative to the number of hosts $\nu$.

[2] T. W. Haynes, S. T. Hedetniemi, and P. J. Slater. Fundamentals of Domination in Graphs. Marcel Dekker, Inc., 1998.

[3] T. Imielinski and H. Korth. Mobile Computing. Kluwer Academic Publishers, 1996.

[4] P. Krishna, M. Chatterjee, N. H. Vaidya, and D. K. Pradhan. A cluster-based approach for routing in ad hoc networks. Proc. of the Second USENIX Symp. on Mobile and Location-Independent Computing, 1995. $1-10$.

[5] C. E. Perkins. Mobile IP: Design Principles and Practices. Addison Wesley, 1997.

[6] C. E. Perkins and E. M. Royer. Highly dynamic destination-sequenced distance-vector routing (DSDV) for mobile computers. Proc. of ACM SIGCOMM'94. 1994, 234-244.

[7] R. Prakash. Unidirectional links prove costly in wireless ad hoc networks. Proc. of the 3rd Int'l Workshop on Discrete Algorithms and Methods for Mobile Computing and Communications. 1999, 15-22.

[8] R. Ramanathan and M. Steenstrup. A survey of routing techniques for mobile communications networks. Mobile Networks and Applications. 1, 1996, 89-104.

[9] E. M. Royer and C. K. Toh. A review of current routing protocols for ad hoc mobile wireless networks. IEEE Personal Communications. Apr. 1999, 46-55.

[10] R. Sivakumar, B. Das, and V. Bharghavan. An improved spine-based infrastructure for routing in ad hoc networks. Proc. of the Int'l Symp. on Computers and Communications (ISCC'98), 1998.

[11] A. Tanenbaum. Computer Networks. Prentice Hall, Inc., 1996.

[12] J. Wu and H. Li. On calculating connected dominating set for efficient routing in ad hoc wireless networks. Proc. of the 3rd Int'l Workshop on Discrete Algorithms and Methods for Mobile Computing and Communications. 1999, 7-14. 